\title{
The conservative treatment of congenital scoliosis with hemivertebra: report of three cases
}

\author{
AG Aulisa ${ }^{*}$, V Guzzanti ${ }^{1}$, C Perisano ${ }^{2}$, G Scudieri ${ }^{2}$, L Bocchino², S Teramo², L Aulisa ${ }^{2}$ \\ From 9th International Conference on Conservative Management of Spinal Deformities - SOSORT 2012 \\ Annual Meeting \\ Milan, Italy. 10-12 May 2012
}

\section{Background}

Scoliosis is the most common congenital disorder. The vertebral disorder can be due to failure of formation, segmentation or their combination. Complete formation failure results in hemivertebra that can cause asymmetrical growth and deformity. The etiopathogenesis of congenital scoliosis is still unclear. Twenty-five percent of congenital curves do not progress, $25 \%$ undergoes mild progression, while the remaining $50 \%$ evolve rapidly and require treatment. Hemivertebra, in the thoraco-lumbar area, display faster rates of progression than those in the lumbar region. The treatment can be either conservative, or surgical. Usually, in rigid and short curves bracing is not recommended, whereas it can be useful for the treatment of secondary curves.

\section{Aim}

To evaluate the efficacy of bracing in congenital scoliosis with hemivertebra.

\section{Methods}

From our database, we identified three patients with congenital scoliosis with hemivertebra. One was 10-year-old at the time of diagnosis and had a hemivertebra localized in L4 with a thoraco-lumbar curve T11-L3. The other one was 6-years-old at the time of diagnosis and had a hemivertebra localized in L2, with a thoraco-lumbar curve T11-L4. The last one was 4-years-old at the time of diagnosis and had a hemivertebra localized in L2, with a thoraco-lumbar curve T11-L3.

\section{Results}

The first patient was treated with a Milwaukee brace, the second with a Boston brace and the third with a

${ }^{1}$ Orthopaedic Department, Children's Hospital Bambino Gesù, Rome, Italy

Full list of author information is available at the end of the article
PASB brace. The Cobb angles at the beginning were $23^{\circ}, 53^{\circ}$ and $25^{\circ}$, respectively. At the end of treatment, the Cobb angles were $18^{\circ}, 33^{\circ}$ and $11^{\circ}$, respectively. At 2 years of follow-up, the curves were $20^{\circ}, 35^{\circ}$ and $13^{\circ}$ degrees, respectively.

\section{Conclusions}

Conservative treatment can be considered a valid means to treat not only the patients with congenital scoliosis with hemivertebra who refuse surgery.

\section{Author details}

'Orthopaedic Department, Children's Hospital Bambino Gesù, Rome, Italy.

${ }^{2}$ Department of Orthopaedics, University Hospital "Agostino Gemelli",

Catholic University of the Sacred Heart, Rome, Italy.

Published: 3 June 2013

\section{References}

1. Kaspiris A, Grivas TB, Weiss HR, Turnbull D: Surgical and conservative treatment of patients with congenital scoliosis: alpha search for longterm results. Scoliosis 6:12.

2. Kaspiris A, Grivas TB, Weiss HR: Congenital scoliosis in monozygotic twins: case report and review of possible factors contributing to its development. Scoliosis 2008, 3:17.

3. Hensinger RN: Congenital scoliosis: etiology and associations. Spine (Phila Pa 1976) 2009, 34(17):1745-1750.

doi:10.1186/1748-7161-8-S1-051

Cite this article as: Aulisa et al:: The conservative treatment of congenital scoliosis with hemivertebra: report of three cases. Scoliosis 2013 8(Suppl 1):051. 